\title{
Altered Insula Activity during Visceral Interoception in Weight-Restored Patients with Anorexia Nervosa
}

\author{
Kara L Kerr ${ }^{1,2}$, Scott E Moseman ${ }^{3}$, Jason A Avery', Jerzy Bodurka ${ }^{1,4,5}$, Nancy L Zucker ${ }^{6}$ and \\ W Kyle Simmons*,1,7
}

'Laureate Institute for Brain Research, Tulsa, Oklahoma, USA; ${ }^{2}$ Department of Psychology, University of Tulsa, Tulsa, Oklahoma, USA; ${ }^{3}$ Laureate Psychiatric Clinic and Hospital, Tulsa, Oklahoma, USA; ${ }^{4}$ College of Engineering, University of Oklahoma, Norman, Oklahoma, USA; ${ }^{5}$ Center for Biomedical Engineering, University of Oklahoma, Norman, Oklahoma, USA; 'SChool of Medicine, Duke University, Durham, North Carolina, USA; ${ }^{7}$ Faculty of Community Medicine, University of Tulsa, Tulsa, Oklahoma, USA

\begin{abstract}
Anorexia nervosa (AN) is a devastating psychiatric illness that is associated with significant morbidity and mortality. Aberrant visceral interoceptive processing within the insula has been hypothesized to be an important mechanism in AN's pathophysiology due to the theoretical link between interoception and emotional experience. We therefore utilized functional magnetic resonance imaging (fMRI) to examine whether altered insula functioning underlies visceral interoception in AN. Fifteen females with restricting-type AN and I5 healthy control females underwent $\mathrm{AMRI}$ while performing an interoceptive attention task during which they focused on sensations in their heart, stomach, and bladder. Participants also performed an anxious rumination task while in the scanner. AN participants were weight-restored and free of psychotropic medications. Two distinct regions of the insula-anterior insula and dorsal mid-insula — exhibited a significant $(p<0.05)$ interaction between group and interoceptive modality. The post hoc analyses revealed that in the dorsal mid-insula the interaction was driven by group differences during stomach interoception ( $p=0.002$, Bonferroni corrected), whereas in the anterior insula the interaction was driven by group differences during heart interoception ( $p=0.03$, Bonferroni corrected). In addition, individuals with AN displayed increased activation during anxious rumination in the dorsal mid-insula, and activation in this region during stomach interoception was correlated with measures of anxiety and psychopathology. This relationship between altered visceral interoception and clinical symptoms in AN suggests an important mechanism for the disorder. Additional research is needed to examine whether interventions targeting visceral interoception may increase the efficacy of treatments for AN.

Neuropsychopharmacology (2016) 4I, 52 I-528; doi: I 0.1038/npp.20I5.174; published online 22 July 2015
\end{abstract}

\section{INTRODUCTION}

Visceral interoception refers to the perception and integration in the brain of afferent (primarily vagal) signals pertaining to the homeostatic state of the body (Craig, 2002). Neuroanatomical and functional neuroimaging evidence points toward a central role for the insular cortex in the integration and representation of these interoceptive signals (Craig, 2002; Khalsa et al, 2009; Kurth et al, 2010; Simmons et al, 2013). For example, attending to interoceptive sensations activates the region of the dorsal midinsular cortex near the terminus of vagal afferent projections from the viscera (Craig, 2002; Farb et al, 2012; Pollatos et al, 2007; Simmons et al, 2013), and lesions to the insula profoundly disrupt interoceptive awareness (Grossi et al, 2014; Khalsa et al, 2009). Dating back to the dawn of modern

*Correspondence: Dr WK Simmons, Simmons Lab, Laureate Institute for Brain Research, 6655 South Yale Avenue, Tulsa, Oklahoma 74I363326, USA, Tel: +| 918502 5106, Fax: +| 9185025135 ,

E-mail:wksimmons@laureateinstitute.org

Received 12 January 2015; revised 15 May 2015; accepted 20 May 2015; accepted article preview online 18 June 2015 psychology, theories have ascribed an important role for the physiological state of the body in the experience of emotion (Damasio, 1994; James, 1890), with more recent accounts highlighting the insula's role in interoception as the link between the body's autonomic state and the subjective experience of emotion, particularly fear and anxiety (Craig, 2002; Paulus and Stein, 2006). For example, according to one account (Paulus and Stein, 2006), 'noisy' interoceptive representation in the insula interferes with the ability to accurately predict the interoceptive consequences of external stimuli on one's internal milieu, resulting in heightened anxiety and withdrawal behaviors. This idea is particularly applicable to anorexia nervosa (AN), a devastating condition with the highest mortality rate of any psychiatric illness (Harris and Barraclough, 1998). AN is associated with somatic preoccupation with visceral gut sensations (eg, fullness; Perez et al, 2013), as well as heightened fear and anxious rumination (Frank et al, 2012; Startup et al, 2013), and enduring anxiety-related personality traits such as harm avoidance (Brewerton et al, 1993; Fassino et al, 2002). Because of this, influential theoretical accounts about the neurobiology of AN have recently highlighted the likely role of interoceptive regions in the insula in the pathophysiology 
of the disorder (Kaye et al, 2009, 2013; Nunn et al, 2011). Although this accords with many of the disorder's clinical features, including AN patients' pathological lack of response to, or inability to detect, homeostatic signals that drive food consumption behaviors, there has heretofore been no experimental evidence demonstrating insular involvement in altered visceral interoception in AN.

To address this gap in the literature, we asked participants with $\mathrm{AN}$ and healthy control (HC) participants to undergo functional magnetic resonance imaging (fMRI) while performing a well-validated interoceptive attention task in which participants focus on the sensations in specific parts of their bodies (Avery et al, 2013; Simmons et al, 2013). Given that many of the most influential theories of emotion ground at least some aspects of emotional experience (particularly negative affect such as anxiety) in visceral sensation, and many patients with AN report heightened visceral/somatic complaints as well as elevated rates of anxiety, we hypothesized that visceral interoception and anxiety in AN might be related. The fMRI task therefore also included anxious rumination trials during which participants worried about specified anxiety-provoking topics in order to examine group differences in regions found to have abnormal activation during interoception in AN patients. The study's methods and analyses aimed to answer two important questions. First, is AN associated with aberrant insula activity during interoceptive attention, and if so, are there differences among visceral targets of interoceptive attention? Second, are individual differences in AN clinical symptomatology related to individual differences in interoceptive processing?

\section{MATERIALS AND METHODS}

\section{Participants}

A total of 15 females (mean body mass index $(B M I)=19.8$, range $=18.6-22.7)$ with restricting-type $\mathrm{AN}$ and $15 \mathrm{HC}$ females (mean $\mathrm{BMI}=20.8$, range $=19.0-23.0)$ with no history of psychiatric disorder participated in this study. Groups did not differ in age (AN mean $=17$ years, range $=13-24$ years; $\mathrm{HC}$ mean $=18$ years, range $=13-23$ years; $t=-1.08, p=0.29$ ). Clinical assessment scores for participants are displayed in Supplementary Table S1. AN participants were scanned after weight restoration to a BMI of at least 18.5 in order to control for the physiological effects of starvation. Most AN participants $(n=11)$ were scanned before discharge from inpatient treatment. All participants were free of psychotropic medication use within the 3 weeks before scanning (6 weeks for fluoxetine). All procedures were approved by the institute's designated institutional review board. Before participation, adult participants provided written informed consent, and adolescent participants provided written informed assent with written informed consent provided by a parent. Participants were provided financial compensation for participation. Please see Supplementary Information for details regarding additional exclusion criteria.

\section{Behavioral Measures}

Participants completed a battery of assessment measures, including the Eating Disorders Inventory-3 (EDI-3),
State-Trait Anxiety Inventory (STAI; Spielberger et al, 1970) Hamilton Anxiety Scale (SIGH-A; Shear et al, 2001) Toronto Alexithymia Scale (TAS; Taylor et al, 1992), and Temperament and Character Inventory (TCI; Cloninger et al, 1994; Luby et al, 1999). Please see Supplementary Information for details regarding these measures and diagnostic procedures.

\section{Task}

The fMRI task consisted of three different event types: exteroception baseline, interoceptive attention, and anxious rumination (Figure 1). Fixation marks were displayed for variable interstimulus intervals lasting $2.5-15 \mathrm{~s}$. The three event types were optimized for fMRI analysis by Optseq2 (http://surfer.nmr.mgh.harvard.edu/optseq/) and presented in pseudorandom order. Visual stimuli were controlled using Eprime2 software (www.pstnet.com). Participants viewed these stimuli through a mirror system mounted on the head coil and back-projected to a screen in the scanner bore. Each scan session contained three runs of this task.

During interoceptive attention (IA) trials, participants viewed the word 'HEART,' 'STOMACH,' or 'BLADDER' for $10 \mathrm{~s}$. Participants were instructed to attend to the intensity of sensations from that organ, such as heartbeat or stomach distension. Past research has demonstrated that focal attention on a perceptual modality amplifies activity in brain regions underlying that modality (Jancke et al, 1999; Johansen-Berg et al, 2000; Somers et al, 1999). This interoceptive attention task capitalizes on this effect as participants focus on naturally occurring interoceptive sensations. We have previously demonstrated that this task

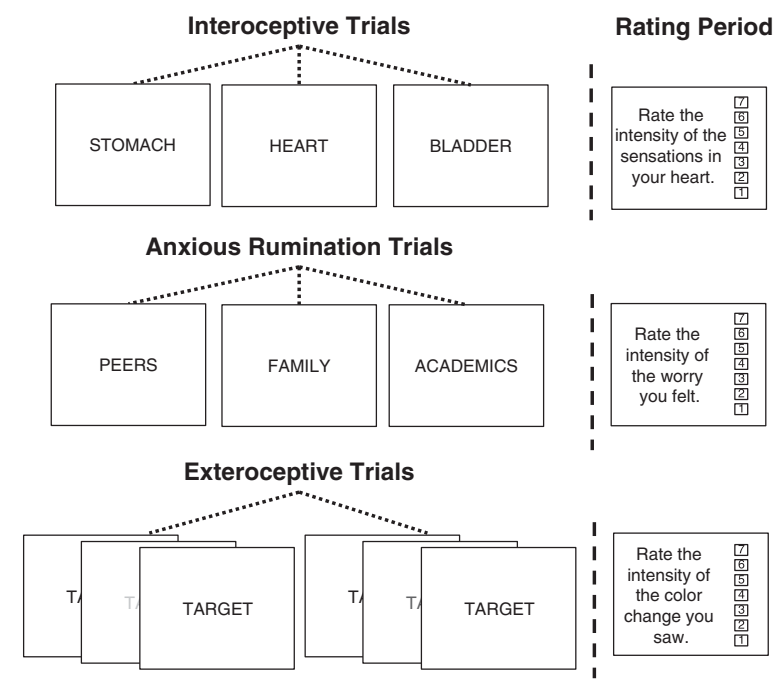

Figure I Focused awareness task design. The focused awareness task consisted of three types of trials: interoceptive attention (IA), anxious rumination trials, and exteroceptive trials. During IA trials, participants focused on the body part indicated by the word on the screen (stomach, heart, or bladder). During anxious rumination trials, participants ruminated about worrisome situations related to the word presented on the screen (peers, family, or academics). Exteroceptive trials were used as a baseline condition for the interoceptive trials. During exteroceptive trials, the font color of the word 'TARGET' repeatedly changed to a lighter shade of gray, and participants focused on the intensity of the color change. Half of the trials in each condition were followed by a rating scale on which participants rated their experience (intensity of bodily sensations, anxious rumination, or color change) during that trial. 
is effective at mapping interoceptive regions in the insula in healthy adults (Simmons et al, 2013). Each word was presented 6 times per scanning run, for a total of 18 interoceptive events per run. Immediately following half of the events ( 3 per modality), participants rated the intensity of the sensations they experienced in the specified body part on a 7-point scale using an MR-compatible scroll wheel.

The exteroceptive baseline condition served as an exteroceptive control for the IA condition. During these trials, the word 'TARGET' was presented on the screen for $10 \mathrm{~s}$. While the word was on the screen, the intensity of the shading of the font would change once per second to a shade of gray that varied across (but not within) trials. Participants were instructed to attend to the intensity of the color change. The exteroceptive baseline condition was presented six times per scanning run. Following half of these trials, a rating scale was presented on the screen on which participants were asked to rate the intensity of the color change they saw.

The anxious rumination condition occurred 6 times per scanning run. Participants were shown the words 'PEERS,' 'FAMILY,' and 'ACADEMICS/CAREER' and instructed to think about situations associated with the presented word that worry them. Immediately following half of the rumination events, participants rated the intensity of their worry during that trial on a 7-point scale.

\section{MRI Data Acquisition}

Functional and structural brain images were acquired using a General Electric Discovery MR750 whole-body 3 Tesla MRI scanner. MRI signal reception utilized a receive-only 32element surface coil head array optimized for parallel imaging (Nova Medical). A single-shot gradient-recalled EPI sequence with Sensitivity Encoding (SENSE) was employed for blood oxygenation level-dependent (BOLD) fMRI scans. For an anatomical reference for the fMRI analyses, a T1-weighted MRI scan with magnetization-prepared rapid gradient echo (MPRAGE) sequence with SENSE was obtained.

\section{Imaging Parameters}

The following EPI imaging parameters were used for this study: FOV/slice/gap $=240 / 2.9 / 0 \mathrm{~mm}, 46$ axial slices per volume, acquisition matrix $=96 \times 96$, repetition/echo time $\mathrm{TR} / \mathrm{TE}=2500 / 30 \mathrm{~ms}$, SENSE acceleration factor $R=2$ in the phase encoding (anterior-posterior) direction, flip angle $=$ $90^{\circ}$, sampling bandwidth $=250 \mathrm{kHz}$, number of volumes 220, scan time $9 \mathrm{~min}$ and $10 \mathrm{~s}$. EPI images were reconstructed into a $128 \times 128$ matrix, with an fMRI voxel volume of $1.875 \times 1.875 \times 2.9 \mathrm{~mm}^{3}$.

Scan parameters for this anatomical scan were $\mathrm{FOV}=240$ $\mathrm{mm}$, axial slices per volume $=130$, slice thickness $=1.1 \mathrm{~mm}$, image matrix $=256 \times 256$, voxel volume $0.938 \times 0.938 \times$ $1.1 \mathrm{~mm}^{3}, \mathrm{TR} / \mathrm{TE}=5 / 1.948 \mathrm{~ms}$, SENSE acceleration factor $R=2$, flip angle $=8^{\circ}$, inversion time $\mathrm{TI}=725 \mathrm{~ms}$, sampling bandwidth $=31.25 \mathrm{kHz}$, scan time $=5 \mathrm{~min}$ and $5 \mathrm{~s}$.

\section{Data Preprocessing and Analysis}

Preprocessing of fMRI data was performed using AFNI (http://afni.nimh.nih.gov/afni). The anatomical scan was registered to the first volume of the EPI data using anatomical-to-epi alignment procedure, and then spatially transformed to the stereotaxic array of Talairach and Tournoux (1988) using automated algorithm in AFNI. The first four volumes of each voxel's time course were excluded from analysis to allow the fMRI signal to reach steady state. Motion correction and spatial transformation were implemented in a single image transformation. The EPI data were resampled to a $1.75 \times 1.75 \times 1.75 \mathrm{~mm}$ grid and smoothed using a $6 \mathrm{~mm}$ full-width at half-maximum Gaussian kernel. Each voxel's mean signal across the time course was then used to normalize the signal value for each EPI volume to percent signal change.

\section{Statistical Analyses}

Data at the participant level were analyzed using a multiple linear regression model. Regressors of interest included each of the interoceptive modalities (heart, stomach, and bladder), the anxious rumination condition, the exteroceptive baseline condition, and the five different types of response periods (when participants were making ratings). Each of the 10 task regressors were constructed by convolution of a gammavariate function and a box-car function with a 10-s width beginning at the onset of each event in order to account for the shape and delay of the BOLD response. Regressors of noninterest to account for each run's signal mean, linear, quadratic, and cubic signal trends were included in the regression model along with six motion parameters (three translations and three rotations) computed during the image registration preprocessing.

The AFNI program 3dANOVA3 was used to evaluate the main effects of group (AN or $\mathrm{HC}$ ) and interoceptive modality (heart, stomach, or bladder) and their interaction. For the IA condition, all subject-level $\beta$ s represent the signal change from the exteroceptive baseline condition. We identified the insula as an a priori region of interest. Masks of the insula in each brain hemisphere were anatomically defined using the TT-N27 atlas brain within AFNI. A voxelwise threshold of $p<0.005$ was set within the insula, and a voxel-wise threshold of $p<0.001$ was set for the rest of the brain. All results were then corrected for multiple comparisons at a cluster-size threshold of $p<0.05$ with the AFNI program 3dClustSim. The post hoc ROI analyses were performed using Bonferroni corrections for multiple comparisons to determine the simple effects for clusters exhibiting significant group $\times$ interoceptive modality interactions within the insula.

We next sought to determine whether the insula regions with a significant interaction during IA also displayed altered activity during anxious rumination. We used the AFNI program 3dROIstats to calculate the average $\beta$ for each participant in each region for the rumination condition and for each interoceptive modality. We then performed $t$-tests to examine group differences in activity during anxious rumination in these regions.

Finally, we sought to determine whether activation during interoception was related to clinical symptomatology. We therefore correlated the average IA $\beta \mathrm{s}$ in the two insular regions exhibiting significant group $\times$ interoceptive modality interactions with the following clinical questionnaire scores: EDI-3 General Psychological Maladjustment Composite (EDI-GPMC), EDI-3 Eating Disorder Risk Composite 
(EDI-EDRC), EDI-3 Interoceptive Deficits (EDI-ID), STAI state anxiety, STAI trait anxiety, SIGH-A, TAS Difficulty Identifying Feelings (TAS-F1), and TCI Harm Avoidance (TCI-HA). Correlations were Bonferroni corrected to control for family-wise error.

\section{RESULTS}

We observed interactions between group and interoceptive modality within two separate regions of the insula-the left dorsal mid-insula and right anterior insula (Figure 2). Five regions located outside of the insula also exhibited a significant interaction effect (Table 1). The right precuneus $(x=6, y=-55$, $z=48$; peak $\mathrm{F}=27.1$; volume $=659 \mathrm{~mm}^{3}$ ) was the only region with a main effect of group; HC participants had greater average activity in the precuneus across interoceptive modalities than did AN participants. See Supplementary Table S2 for regions with a main effect of interoceptive modality.
The post hoc analyses of the simple effects from the ANOVA revealed that AN participants exhibited decreased activity during stomach IA in the left dorsal mid-insula compared with HC participants $(t=-3.81, \mathrm{df}=28, p=0.002$, Bonferroni corrected). In contrast, in the right anterior insula, participants with AN exhibited greater activity during heart IA than did HC participants $(t=2.75, \mathrm{df}=28, p=0.03$, Bonferroni corrected). There were no statistically significant simple effects in any of the noninsular regions after Bonferroni corrections. Because the AN participants reported higher levels of anxiety, we conducted analyses to determine whether the interoception effects were mediated by self-report and clinician measures of anxiety (SIGH-A, STAI trait, STAI state, and anxious rumination intensity ratings during the scan). None of these measures mediated the effects of group differences on interoceptive activity in either of the insula regions.

We next performed an ROI analysis of brain activation during anxious rumination in the insula regions

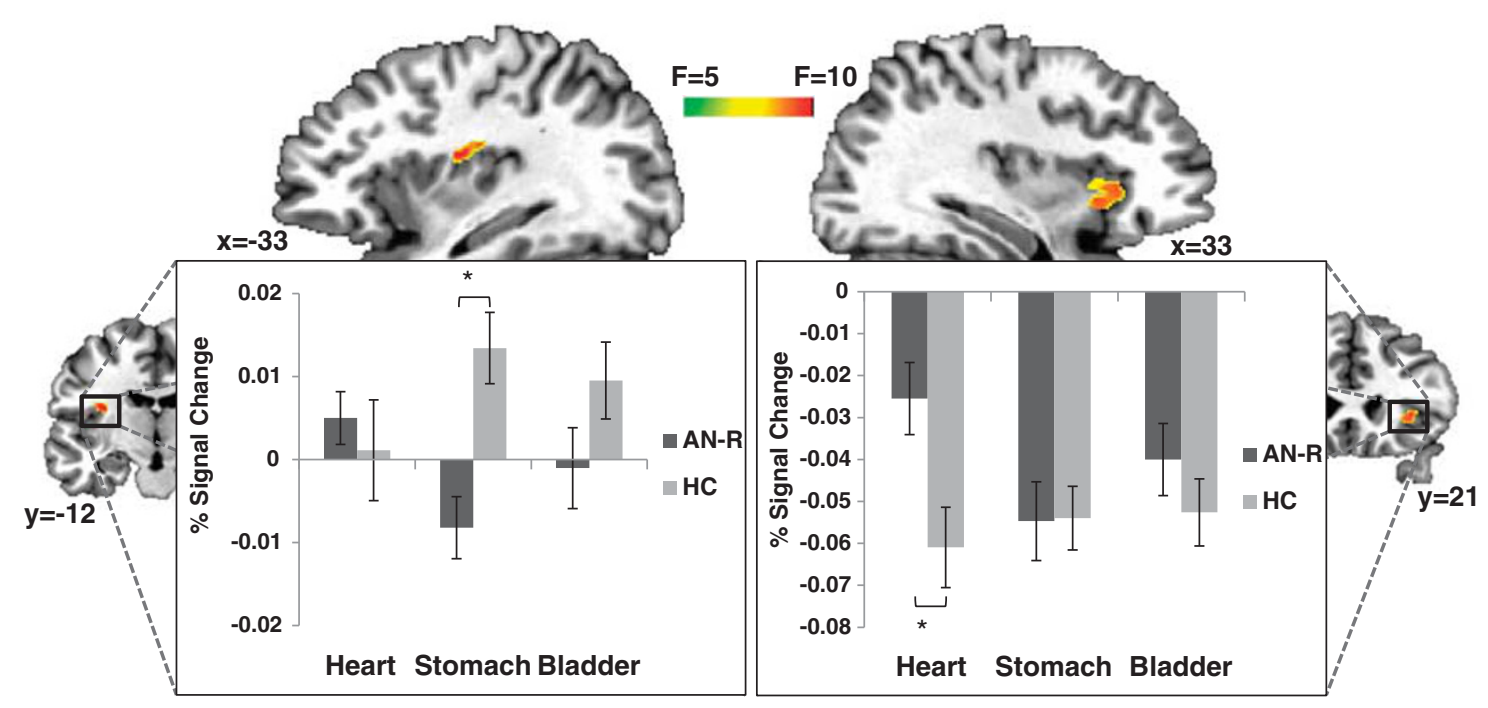

Figure 2 Altered insula activity during visceral interoception in patients with anorexia nervosa. A region-of-interest analysis (voxel-wise threshold $p<0.005$, cluster size corrected for multiple comparisons at $p<0.05$ ) revealed two separate insula regions - the dorsal mid-insula and anterior insula- that exhibited a significant Group (AN, HC) $\times$ Interoceptive Modality (heart, stomach, bladder) interaction. The post hoc simple effects analyses revealed that the interaction in the dorsal mid-insula was because of group differences during stomach interoception $(p<0.002$, Bonferroni corrected), whereas in the anterior insula the interaction resulted from group differences during heart interoception ( ${ }^{2}<<0.03$, Bonferroni corrected).

Table I Regions Exhibiting a Group × Interoceptive Modality Interaction

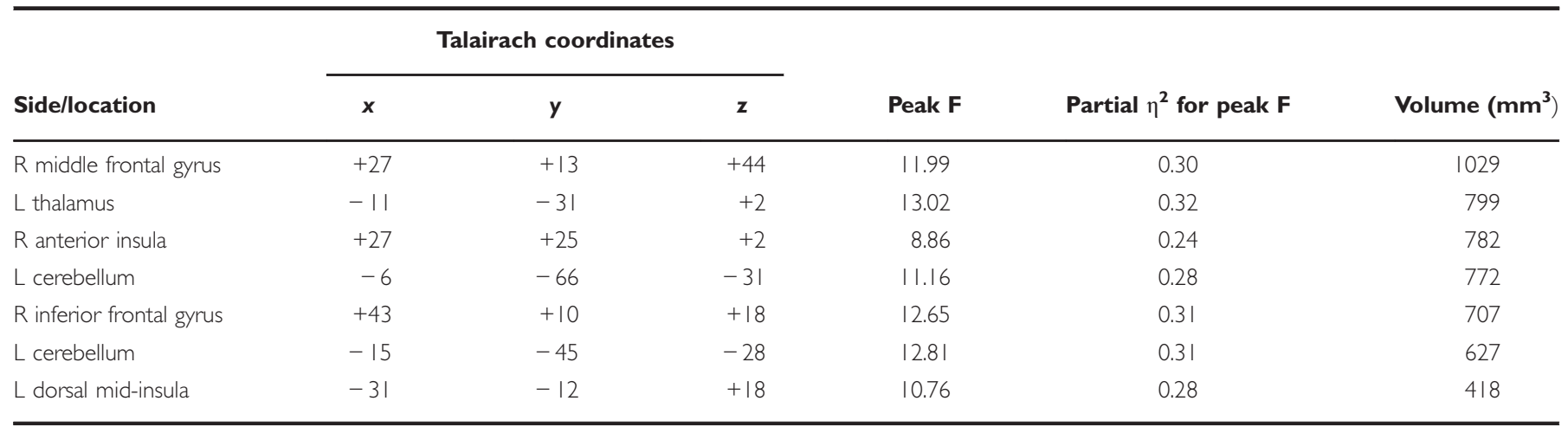

A voxel-wise threshold of $p<0.005$ was set for the insula regions of interest, with a voxel-wise threshold of $p<0.00$ I for the rest of the brain. All significant activations passed cluster size correction for multiple comparisons at $p<0.05$. 


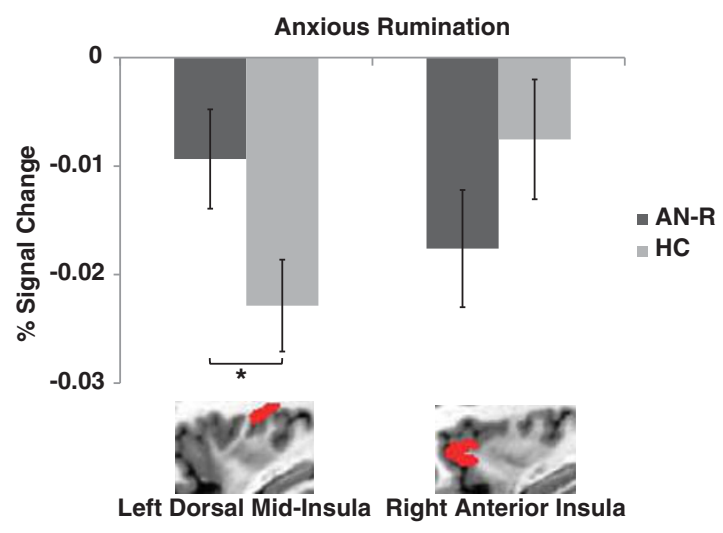

Figure 3 Anorexia nervosa is associated with aberrant activation in the dorsal mid-insula during anxious rumination. A region-of-interest analysis of activation during anxious rumination in the two insula clusters that exhibited an interaction during visceral interoception revealed significant group differences in the dorsal mid-insula $(* p<0.04)$ but not the anterior insula $(p=0.20)$.

demonstrating an interaction between group and interoceptive modality. The ROI analysis (Figure 3) revealed a significant group difference between $\mathrm{AN}$ and $\mathrm{HC}$ participants during anxious rumination in the dorsal mid-insula $(p<0.04)$, but not in the anterior insula $(p=0.20)$.

Finally, in order to determine the relationship between our neuroimaging findings and clinical symptoms of $\mathrm{AN}$, we assessed the correlation between clinical measures of anxiety and eating disorder psychopathology with activity in the two insula regions with altered interoceptive activity in AN participants. After Bonferroni corrections for multiple comparisons, significant negative correlations were found between activity during stomach IA in the dorsal mid-insula and scores on clinical measures of anxiety, harm avoidance, alexithymia, psychological maladjustment, and interoceptive deficits (EDI-GPMC, EDI-ID, STAI state anxiety, STAI trait anxiety, SIGH-A, TAS-F1, and TCI-HA; see Table 2 and Supplementary Figure S1). These findings appear to be remarkably specific to stomach IA and the dorsal mid-insula, as no significant correlations were observed for the other interoceptive modalities in either insula ROI.

\section{DISCUSSION}

Our findings provide the first experimental evidence that AN is associated with abnormal visceral interoceptive activity in the insula. Importantly, decreased activity in the dorsal midinsula during gastric interoception may contribute to $\mathrm{AN}$ patients' anxious temperament, as demonstrated by heightened activity in this region during anxious rumination. Although AN participants exhibited increased activity in the anterior insula during cardiac interoception, this activity was not related to their clinical symptom presentation. In contrast, individual differences in dorsal mid-insula activity during stomach interoception were associated with participants' anxiety and eating disorder psychopathology. Together, these findings suggest that aberrant interoceptive activity within the dorsal mid-insula is an important contributor to the pathophysiology of AN.
Table 2 Relationships between Insular Activity and Symptom Measures across Participants

\begin{tabular}{|c|c|c|c|c|c|c|}
\hline \multirow[b]{2}{*}{$\begin{array}{l}\text { Clinical } \\
\text { measures }\end{array}$} & \multicolumn{3}{|c|}{ Left dorsal mid-insula } & \multicolumn{3}{|c|}{ Right anterior insula } \\
\hline & Heart & Stomach & Bladder & Heart & Stomach & Bladder \\
\hline STAl-state & 0.01 & $-0.54^{\mathrm{a}}$ & -0.19 & 0.27 & 0.05 & 0.22 \\
\hline STAl-trait & 0.05 & $-0.58^{\mathrm{a}}$ & -0.26 & 0.40 & 0.10 & 0.33 \\
\hline SIGH-A & 0.08 & $-0.52^{\mathrm{a}}$ & -0.37 & 0.40 & 0.06 & 0.23 \\
\hline $\mathrm{TCl}-\mathrm{HA}$ & -0.12 & $-0.54^{\mathrm{a}}$ & -0.36 & 0.27 & 0.05 & 0.33 \\
\hline TAS-FI & 0.08 & $-0.65^{\mathrm{a}}$ & -0.21 & 0.19 & -0.10 & 0.14 \\
\hline EDI-ID & 0.05 & $-0.59^{\mathrm{a}}$ & -0.21 & 0.14 & -0.05 & 0.06 \\
\hline EDI-GPMC & 0.04 & $-0.60^{\mathrm{a}}$ & -0.28 & 0.25 & -0.00 & 0.21 \\
\hline EDI-EDRC & 0.13 & -0.45 & -0.23 & 0.27 & -0.05 & 0.10 \\
\hline
\end{tabular}

Abbreviations: EDI-ID, Eating Disorders Inventory-3 Interoceptive Deficits; EDI-EDRC, Eating Disorders Inventory-3 Eating Disorder Risk Composite; EDI-GPMC, Eating Disorders Inventory-3 General Psychological Maladjustment; STAl-state, State-Trait Anxiety Inventory-state anxiety; STAI-trait, State-Trait Anxiety Inventory-trait anxiety; SIGH-A, Hamilton Anxiety Scale; TCl-HA,

Temperament and Character Inventory Harm Avoidance; TAS-FI, Toronto Alexithymia Scale Factor I (difficulty identifying feelings).

${ }^{\mathrm{a}} P<0.05$ after Bonferroni corrections for multiple comparisons.

In addition to abnormal insula activity, individuals with AN exhibited decreased activity in the precuneus across interoceptive modalities. Although we did not hypothesize that there would be group differences in this region, this finding accords with past research on the precuneus in both healthy individuals and those with $\mathrm{AN}$. The precuneus is involved in mental self-representations, including mental imagery of the self (Cavanna and Trimble, 2006), and the linking of interoceptive and contextual information to influence emotional experience (Terasawa et al, 2013). In addition, individuals with AN have demonstrated deactivation of the precuneus when viewing pictures of themselves (Sachdev et al, 2008), and in one study a small sample of AN participants continued to show deficits in gray matter in the precuneus after recovery (Joos et al, 2011). Thus, altered interoceptive processing in AN does not appear to be limited to the insula. Further research should examine the role of the precuneus in this disorder, particularly as it relates to selfreferential and emotional experience.

While group differences in the precuneus occurred across interoceptive modalities, the anterior and dorsal mid-insula exhibited group differences only during heart or stomach interoception, respectively. Past studies have illustrated that functional differentiation exists within the insula, with the anterior insula playing a role in the representation of negative emotions, and the middle and posterior insula being more involved in the processing of internal physiological sensations (Craig, 2002; Kurth et al, 2010; Simmons et al, 2013). Evidence suggests that the anterior insula particularly underlies anxiety (Paulus et al, 2003; Servaas et al, 2014; Simmons et al, 2013), possibly because of its involvement in anticipating the interoceptive consequences of external stimuli (Paulus and Stein, 2006).

Given this literature base, in addition to previous findings and theories suggesting the importance of the anterior insula in AN (Kaye et al, 2009, 2013; Nunn et al, 2011; Oberndorfer 
et al, 2013; Strigo et al, 2013), it is interesting that the group differences found in this area occurred during cardiac IA, not anxious rumination. Past evidence indicates the anterior insula may underlie interoceptive prediction (Barrett and Simmons, 2015). This, in conjunction with poor heartbeat perception in AN (Pollatos et al, 2008), suggests that AN participants' heightened anterior insula activity during heart IA may result from alterations in the cardiac prediction signal.

This study adds to a growing body of evidence that AN is associated with altered interoception. For example, AN is associated with reduced heartbeat perception in the ill state (Pollatos et al, 2008) and a dissociation between reported emotional experience and physiological measures (ie, skin conductance response; Nandrino et al, 2012). These findings are generally consistent with a prior fMRI study demonstrating decreased insula activity during pain perception (Strigo et al, 2013). Indeed, a recent influential account of the neurobiology of AN anticipated the present findings (Kaye et al, 2009), even though at the time of that account there was no direct experimental evidence for the insula's role in aberrant visceral interoception in AN (Kaye et al, 2009). The present study is the first to provide direct experimental evidence to this effect, thereby strongly supporting this theoretical account.

A thorough interpretation of our results must take into consideration that it was only during gastric IA that $\mathrm{AN}$ participants displayed altered functioning of the interoceptive cortex located in the dorsal mid-insula. Taken together with evidence for gastrointestinal complaints in individuals with AN (Boyd et al, 2005; Hadley and Walsh, 2003; Waldholtz and Andersen, 1990), this suggests that individuals with AN may be hypersensitive to, and perhaps constantly monitoring, interoceptive sensations from their gastrointestinal tract. Thus, when they are instructed to focus on these sensations, there is relatively little change from baseline activation in interoceptive cortex. The specificity of aberrant dorsal mid-insular functioning to gastric IA is especially important in light of a previous finding from our laboratory wherein individuals with major depressive disorder demonstrated altered dorsal mid-insula activity during heart, stomach, and bladder IA (Avery et al, 2013). In conjunction with the findings of the present study, this suggests different psychiatric disorders are associated with variable profiles of altered interoception.

Importantly, activation in the dorsal mid-insula during gastric IA was negatively correlated with clinical symptoms, including anxiety and harm avoidance. This links gastric interoception and anxiety in $\mathrm{AN}$, and is supported by theories emphasizing the relationship between interoception and emotional experience (Barrett et al, 2007; Craig, 2002; James, $1890)$. Based on the theory that anxiety results from an exaggerated interoceptive signal predicting the effect of a stimulus on one's physiology (Paulus and Stein, 2006), a similar process may be occurring in $\mathrm{AN}$ that is specific to gastric interoception. For instance, hypersensitivity to sensations from the gastrointestinal tract might lead to anxiety in the presence of food as it becomes predictive of an aversive gastrointestinal response (eg, bloating, fullness). This would then lead to the restrictive eating behavior that constitutes the core pathology of AN.

This is the first study to demonstrate altered insular function during attention to visceral interoceptive sensations in $\mathrm{AN}$ and to link this function to clinical symptoms such as anxiety. Although this study has many strengths, including a sample of unmedicated, weight-restored patients, one limitation is the inability to monitor the accuracy of the participants' interoceptive attention. Mechanisms through which performance could be measured, however, would necessarily require participants to simultaneously report certain parameters (eg, heart rate) that would likely interfere with the interoceptive nature of the task. This would require exteroceptive processes for reporting, in addition to the likelihood of disproportionately increasing anxiety in the AN patients because of their high levels of perfectionism. Thus, the methods used in this study were well suited for answering the research question of possible differences in interoceptive attention and experience, rather than accuracy, and have been used in other previously published studies of interoception (Avery et al, 2013; Simmons et al, 2013).

Our findings support the theory that aberrant functioning of interoceptive cortex in the dorsal mid-insula during gastric interoception contributes to the symptomatology of $\mathrm{AN}$, including anxiety. This accords with previous research that comorbid anxiety in $\mathrm{AN}$ is associated with increased gastrointestinal complaints (Boyd et al, 2005), that sensitivity to sensation is associated with body image disturbance in AN (Zucker et al, 2013), and that sensory sensitivity is related to emotional dysregulation in AN (Merwin et al, 2013). Our findings have important implications for both understanding the etiology of AN and informing its treatment. First, AN may partially result from visceral hypersensitivity that leads to an association between food and gastric discomfort, which contributes to anxiety. Second, although it is generally recognized that patients often have an increase in gastrointestinal symptoms during refeeding, this study suggests that alterations in stomach interoception might exacerbate these symptoms and influence psychological disturbances, particularly anxiety. This could manifest as a vicious cycle during the weight restoration process, with gastric discomfort leading to increased anxiety that then leads to greater gastric discomfort. Cognitive approaches to therapy, including acceptance and commitment therapy, directed at interoceptive signals such as fullness and gastrointestinal symptoms, may therefore be beneficial in the treatment of AN. Previous studies with phobic individuals have found changes in insula activation following treatment with cognitive-behavioral therapy (Hauner et al, 2012). Medications targeting gastrointestinal symptoms, particularly during refeeding, may contribute to the alleviation of anxiety as well. Our results also indicate that outcome measures in clinical trials for AN should examine the effects of treatment on gastric interoception and anxiety. Future research should further elucidate the role of gastric interoception in the pathophysiology of $\mathrm{AN}$ by studying its effect on responses to food stimuli and eating behavior, as well as developing treatments that incorporate empirical evidence of the relationship between gastric interoception and anxiety.

\section{FUNDING AND DISCLOSURE}

This research was supported by a NARSAD Young Investigator Award to WKS, a NIMH grant (K01MH096175-01) to WKS, the Oklahoma Center for the Advancement of Science and Technology (Grant HR10-141), the Laureate Institute for Brain Research, and the William K. Warren Foundation. The authors declare no conflict of interest. 


\section{ACKNOWLEDGMENTS}

We thank Danielle DeVille for providing helpful feedback on this manuscript, Joel Barcalow and Jennifer Dobson for conducting participant recruitment and assessment, and the staff of the Laureate Eating Disorders Program for facilitating patient participation in this study. We also thank the research participants and their families for their time and effort.

\section{REFERENCES}

Avery JA, Drevets WC, Moseman SE, Bodurka J, Barcalow JC, Simmons WK (2013). Major depressive disorder is associated with abnormal interoceptive activity and functional connectivity in the insula. Biol Psychiatry 78: 258-266.

Barrett LF, Mesquita B, Ochsner KN, Gross JJ (2007). The experience of emotion. Annu Rev Psychol 58: 373-403.

Barrett LF, Simmons WK (2015). Interoceptive predictions in the brain. Nat Rev Neurosci 16: 419-429.

Boyd C, Abraham S, Kellow J (2005). Psychological features are important predictors of functional gastrointestinal disorders in patients with eating disorders. Scand J Gastroenterol 40: 929-935.

Brewerton TD, Hand LD, Bishop ER (1993). The Tridimensional Personality Questionnaire in eating disorder patients. Int J Eat Disord 14: 213-218.

Cavanna AE, Trimble MR (2006). The precuneus: a review of its functional anatomy and behavioural correlates. Brain 129: $564-583$.

Cloninger CR, Przybeck TR, Svrakic DM, Wetzel RD (1994). The Temperament and Character Inventory: A Guide to its Development and Use. Center for Psychobiology of Personality: St Louis, MO.

Craig AD (2002). How do you feel? Interoception: the sense of the physiological condition of the body. Nat Rev Neurosci 3: 655-666.

Damasio AR (1994). Descartes' Error: Emotion, Reason, and the Human Brain. Putnam: New York, NY.

Farb NAS, Segal ZV, Anderson AK (2012). Attentional modulation of primary interoceptive and exteroceptive cortices. Cereb Cortex 23: $114-126$.

Fassino S, Abbate-Daga G, Amianto F, Leombruni P, Boggio S, Rovera GG (2002). Temperament and character profile of eating disorders: a controlled study with the Temperament and Character Inventory. Int J Eat Disord 32: 412-425.

Frank GKW, Roblek T, Shott ME, Jappe LM, Rollin MDH, Hagman JO et al (2012). Heightened fear of uncertainty in anorexia and bulimia nervosa. Int J Eat Disord 45: 227-232.

Grossi D, De Vita A, Palermo L, Sabatini U, Trojano L, Guariglia C (2014). The brain network for self-feeling: a symptom-lesion mapping study. Neuropsychologia 63C: 92-98.

Hadley SJ, Walsh BT (2003). Gastrointestinal disturbances in anorexia nervosa and bulimia nervosa. Curr Drug Targets CNS Neurol Disord 2: 1-9.

Harris EC, Barraclough B (1998). Excess mortality of mental disorder. Br J Psychiatry 173: 11-53.

Hauner KK, Mineka S, Voss JL, Paller KA (2012). Exposure therapy triggers lasting reorganization of neural fear processing. Proc Natl Acad Sci USA 109: 9203-9208.

James W (1890). Principles of Psychology. Macmillan: London.

Jancke L, Mirzazade S, Shah NJ (1999). Attention modulates activity in the primary and the secondary auditory cortex: a functional magnetic resonance imaging study in human subjects. Neurosci Lett 266: 125-128.

Johansen-Berg H, Christensen V, Woolrich M, Matthews PM (2000). Attention to touch modulates activity in both primary and secondary somatosensory areas. Neuroreport 11: 1237-1241.
Joos A, Hartmann A, Glauche V, Perlov E, Unterbrink T, Saum B et al (2011). Grey matter deficit in long-term recovered anorexia nervosa patients. Eur Eat Disord Rev 19: 59-63.

Kaye WH, Fudge JL, Paulus M (2009). New insights into symptoms and neurocircuit function of anorexia nervosa. Nat Rev Neurosci 10: $573-584$.

Kaye WH, Wierenga CE, Bailer UF, Simmons AN, Bischoff-Grethe A (2013). Nothing tastes as good as skinny feels: the neurobiology of anorexia nervosa. Trends Neurosci 36: 110-120.

Khalsa SS, Rudrauf D, Feinstein JS, Tranel D (2009). The pathways of interoceptive awareness. Nat Neurosci 12: 1494-1496.

Kurth F, Zilles K, Fox PT, Laird AR, Eickhoff SB (2010). A link between the systems: functional differentiation and integration within the human insula revealed by meta-analysis. Brain Struct Funct 214: 519-534.

Luby JL, Svrakic DM, McCallum K, Przybeck TR, Cloninger CR (1999). The Junior Temperament and Character Inventory: preliminary validation of a child self-report measure. Psychol Rep 84: 1127-1138.

Merwin RM, Moskovich AA, Wagner HR, Ritschel LA, Craighead LW, Zucker NL (2013). Emotion regulation difficulties in anorexia nervosa: relationship to self-perceived sensory sensitivity. Cogn Emot 27: 441-452.

Nandrino J-L, Berna G, Hot P, Dodin V, Latrée J, Decharles S et al (2012). Cognitive and physiological dissociations in response to emotional pictures in patients with anorexia. J Psychosom Res 72: $58-64$.

Nunn K, Frampton I, Fuglset TS, Törzsök-Sonnevend M, Lask B (2011). Anorexia nervosa and the insula. Med Hypotheses 76: 353-357.

Oberndorfer TA, Simmons A, McCurdy D, Strigo I, Matthews S, Yang $\mathrm{T}$ et al (2013). Greater anterior insula activation during anticipation of food images in women recovered from anorexia nervosa versus controls. Psychiatry Res Neuroimaging 214: $132-141$.

Paulus MP, Rogalsky C, Simmons A, Feinstein JS, Stein MB (2003). Increased activation in the right insula during risk-taking decision making is related to harm avoidance and neuroticism. Neuroimage 19: 1439-1448.

Paulus MP, Stein MB (2006). An insular view of anxiety. Biol Psychiatry 60: 383-387.

Perez ME, Coley B, Crandall W, Lorenzo C, Di, Bravender T (2013). Effect of nutritional rehabilitation on gastric motility and somatization in adolescents with anorexia. J Pediatr 163: 867-872 e1.

Pollatos O, Kurz A-L, Albrecht J, Schreder T, Kleemann AM, Schöpf V et al (2008). Reduced perception of bodily signals in anorexia nervosa. Eat Behav 9: 381-388.

Pollatos O, Schandry R, Auer DP, Kaufmann C (2007). Brain structures mediating cardiovascular arousal and interoceptive awareness. Brain Res 1141: 178-187.

Sachdev P, Mondraty N, Wen W, Gulliford K (2008). Brains of anorexia nervosa patients process self-images differently from non-self-images: an fMRI study. Neuropsychologia 46: 2161-2168.

Servaas MN, Riese H, Ormel J, Aleman A (2014). The neural correlates of worry in association with individual differences in neuroticism. Hum Brain Mapp 35: 4303-4315.

Shear MK, Bilt J, Vander, Rucci P, Endicott J, Lydiard B, Ottow MW et al (2001). Reliability and validity of a structured interview guide for the Hamilton Anxiety Rating Scale (SIGH-A). Depress Anxiety 13: $166-178$.

Simmons WK, Avery JA, Barcalow JC, Bodurka J, Drevets WC, Bellgowan P (2013). Keeping the body in mind: insula functional organization and functional connectivity integrate interoceptive, exteroceptive, and emotional awareness. Hum Brain Mapp 34: 2944-2958.

Somers DC, Dale AM, Seiffert AE, Tootell RBH (1999). Functional MRI reveals spatially specific attentional modulation in human primary visual cortex. Proc Natl Acad Sci USA 96: $1663-1668$. 
Spielberger CD, Gorsuch RL, Lushene RE (1970). Manual for the State-Trait Anxiety Inventory. Consulting Psychologists Press: Palo Alto, CA.

Startup H, Lavender A, Oldershaw A, Stott R, Tchanturia K, Treasure J et al (2013). Worry and rumination in anorexia nervosa. Behav Cogn Psychother 41: 301-316.

Strigo IA, Matthews SC, Simmons AN, Oberndorfer T, Klabunde M, Reinhardt LE et al (2013). Altered insula activation during pain anticipation in individuals recovered from anorexia nervosa: evidence of interoceptive dysregulation. Int J Eat Disord 46: 23-33.

Talairach J, Tournoux P (1988). Co-Planar Stereotaxic Atlas of the Human Brain. Thieme: New York, NY.
Taylor GJ, Bagby RM, Parker JDA (1992). The Revised Toronto Alexithymia Scale: some reliability, validity, and normative data. Psychother Psychosom 57: 34-41.

Terasawa Y, Fukushima H, Umeda S (2013). How does interoceptive awareness interact with the subjective experience of emotion? An fMRI study. Hum Brain Mapp 34: 598-612.

Waldholtz BD, Andersen AE (1990). Gastrointestinal symptoms in anorexia nervosa: a prospective study. Gastroenterology 98: 1415-1419.

Zucker NL, Merwin RM, Bulik CM, Moskovich A, Wildes JE, Groh J (2013). Subjective experience of sensation in anorexia nervosa. Behav Res Ther 51: 256-265.

Supplementary Information accompanies the paper on the Neuropsychopharmacology website (http://www.nature.com/npp) 